\title{
SEMICLASSICAL LIMIT OF THE NONLINEAR SCHRÖDINGER-POISSON EQUATION WITH SUBCRITICAL INITIAL DATA *
}

\author{
HAILIANG $\mathrm{LIU}^{\dagger}$ AND EITAN TADMOR $\ddagger$
}

\begin{abstract}
We study the semi-classical limit of the nonlinear Schrödinger-Poisson (NLSP) equation for initial data of the WKB type. The semi-classical limit in this case is realized in terms of a density-velocity pair governed by the Euler-Poisson equations. Recently we have shown in [ELT, Indiana Univ. Math. J., 50 (2001), 109-157], that the isotropic Euler-Poisson equations admit a critical threshold phenomena, where initial data in the sub-critical regime give rise to globally smooth solutions. Consequently, we justify the semi-classical limit for sub-critical NLSP initial data and confirm the validity of the WKB method.
\end{abstract}

1. Introduction. Consider the nonlinear Schrödinger-Poisson (NLSP) equation,

$$
i \epsilon \psi_{t}^{\epsilon}=-\frac{\epsilon^{2}}{2} \Delta_{x} \psi^{\epsilon}-k\left(\Delta_{x}^{-1}\left(\left|\psi^{\epsilon}\right|^{2}-c\right)\right) \psi^{\epsilon}, \quad x \in \mathbb{R}^{d}, \quad t \in \mathbb{R}^{+}
$$

subject to the initial condition

$$
\psi_{0}^{\epsilon}(x)=\sqrt{\rho_{0}(x)} e^{i S_{0}(x) / \epsilon}, \quad x \in \mathbb{R}^{d} .
$$

Here, $\psi^{\epsilon}(\cdot, t)$ is a complex-valued wavefunction depending on the scaled Planck constant $\epsilon \sim \hbar$, with $k$ and $c>0$ being scaled physical constants. The NLSP equation (1.1) has been studied in different contexts, and in particular, as the fundamental equation in semiconductors applications, with $c>0$ standing for a constant background charge and $k \sim \lambda^{-2}, \lambda<<1$ being the Debye number. We refer the reader to the recent review $[\mathrm{GLM}]$ and references therein.

The connection between Schrödinger equations and the classical hydrodynamics was already noted in 1927 by Madelung, in the context of semi-classical limit of the Nonlinear Schrödinger (NLS) equation. To this end, one identifies two physically relevant observable quantities - the fluid density $\rho^{\epsilon}:=\left|\psi^{\epsilon}\right|^{2}$, and the fluid velocity $u^{\epsilon}:=\epsilon \nabla_{x} \arg \psi^{\epsilon}$. Indeed, introducing $S^{\epsilon}$ as the phase of the wave function

$$
\psi^{\epsilon}=\sqrt{\rho^{\epsilon}(x, t)} e^{i S^{\epsilon}(x, t) / \epsilon}
$$

and separating real and imaginary parts in the NLSP equation (1.1), one obtains the following irrotational flow equations for the density-velocity pair, $\left(\rho^{\epsilon}, u^{\epsilon}:=\nabla_{x} S^{\epsilon}\right)$, consult e.g., [LL]

$$
\begin{aligned}
\rho_{t}^{\epsilon}+\nabla \cdot\left(\rho^{\epsilon} u^{\epsilon}\right) & =0, \\
u_{t}^{\epsilon}+u^{\epsilon} \cdot \nabla u^{\epsilon} & =k \nabla \Delta_{x}^{-1}\left(\rho^{\epsilon}-c\right)+\frac{\epsilon^{2}}{2} \nabla\left[\frac{\Delta \sqrt{\rho^{\epsilon}}}{\sqrt{\rho^{\epsilon}}}\right] .
\end{aligned}
$$

The resulting system amounts to a dispersive regularization with the square of the Planck constant playing the role of the amplitude of dispersion. If we argue formally

\footnotetext{
${ }^{*}$ Received June 22, 2002; accepted for publication December 18, 2002.

${ }^{\dagger}$ Department of Mathematics, Iowa State University, Ames, IA 50011, USA (hliu@iastate.edu).

¥Department of Mathematics, Center for Scientific Computation And Mathematical Modeling (CSCAMM) and Institute for Physical Science \& Technology (IPST), University of Maryland, College Park, MD 20742-4015, USA (tadmor@cscamm.umd.edu).
} 
that this $\mathcal{O}\left(\epsilon^{2}\right)$ term on the right of (1.4) is negligible as $\epsilon \rightarrow 0$, then the corresponding limiting system becomes the Euler-Poisson equations

$$
\begin{aligned}
\rho_{t}+\nabla \cdot(\rho u) & =0, \\
u_{t}+u \cdot \nabla u & =k \nabla \Delta_{x}^{-1}(\rho-c),
\end{aligned}
$$

subject to given initial data

$$
\rho(x, 0)=\rho_{0}(x), \quad u(x, 0)=\nabla S_{0}(x) .
$$

The dispersive regularization term in (1.4) represents a quantum correction, and the corresponding system (1.3)-(1.4) governing the observables $\left(\rho^{\epsilon}, u^{\epsilon}\right)$ could be viewed as Quantum Euler-Poisson (QEP) system, a specific example to larger class of quantum hydrodynamical systems, [SMM].

The above argument is, of course, only formal. The Madelung's transformation relies on the assumption that the amplitude $\rho^{\epsilon}$ does not vanish and that the phase $S^{\epsilon}$ remains nonsingular, for otherwise the transformation is not well-defined and the equations (1.3), (1.4) become singular, even though NLSP itself is still regular. The challenging issue here is to justify such a dispersive limit. The asymptotics of observable quantities as $\epsilon \rightarrow 0$ is known as 'semi-classical', expressing the passage from quantum to Newton mechanics, where the time and distance scales become large enough relative to Planck's constant. The 'semi-classical' limit problem associated with the NLSP equation (1.1) can be summarized as follows. We consider the family of solutions for the NLSP Cauchy problem (1.1), $\left\{\psi^{\epsilon}, \epsilon>0\right\}$, subject to the WKB-type initial data

$$
\psi^{\epsilon}(x, 0)=\sqrt{\rho_{0}(x)} e^{i S_{0}(x) / \epsilon}, \quad x \in \mathbb{R}^{d}
$$

where $\rho_{0}$ and $S_{0}$ are given smooth functions. The purpose is to determine the behavior of the wavefunction $\psi^{\epsilon}$ as $\epsilon \rightarrow 0$, through the dynamics governing semi-classical limits of its observables, the density, $\rho=\lim \left|\psi^{\epsilon}\right|^{2}$, and the velocity $u=\lim \epsilon \nabla_{x} \arg \psi^{\epsilon}$.

As noted earlier, this argument is self-consistent as long as the solution of the Euler-Poisson system (1.5)-(1.6) remains classical. Motivated by the recent regularity results for general a class of isotropic Euler-Poisson equations, [ELT], we turn to justify the (strong) semi-classical limit of the NLSP. In this paper we restrict our attention to the one-dimensional problem

$$
i \epsilon \psi_{t}^{\epsilon}=-\frac{\epsilon^{2}}{2} \psi_{x x}^{\epsilon}+V^{\epsilon} \psi^{\epsilon}, \quad V_{x x}^{\epsilon}=k\left(\left|\psi^{\epsilon}\right|^{2}-c\right) .
$$

The corresponding one-dimensional QEP system reads

$$
\begin{aligned}
\rho_{t}^{\epsilon}+\left(\rho^{\epsilon} u^{\epsilon}\right)_{x} & =0 \\
u_{t}^{\epsilon}+u^{\epsilon} u_{x}^{\epsilon} & =V_{x}^{\epsilon}+\frac{\epsilon^{2}}{2}\left(\frac{\left(\sqrt{\rho^{\epsilon}}\right)_{x x}}{\sqrt{\rho^{\epsilon}}}\right)_{x}, \quad V_{x x}^{\epsilon}=k\left(\rho^{\epsilon}-c\right),
\end{aligned}
$$

and we are inquiring whether the one-dimensional NLSP (1.8) has a 'faithful' description in terms of its formal semi-classical limit system, the one-dimensional EulerPoisson equations,

$$
\begin{aligned}
\rho_{t}+(\rho u)_{x} & =0, \\
u_{t}+u u_{x} & =V_{x}, \quad V_{x x}=k(\rho-c) .
\end{aligned}
$$


In [ELT] we have shown that the one-dimensional Euler-Poisson system (1.11)(1.12) with initial data, $\left(\rho_{0}, u_{0}\right), \int\left(\rho_{0}(\cdot)-c\right)=0$, below certain critical threshold, i.e.,

$$
\left|\partial_{x} u_{0}\right|<\sqrt{k\left(2 \rho_{0}-c\right)}
$$

retains a globally smooth solution. Equipped with this regularity result for the limiting Euler-Poisson system, it is natural to inquire about the regularity of its dispersive regularization, the QEP equations (1.9)-(1.10). Our main results show the existence of globally smooth solution, $\psi^{\epsilon}$, of (1.8), which assume sub-critical smooth initial data, (1.7),

$$
\left|\partial_{x}^{2} S_{0}\right|<\sqrt{k\left(2\left|\psi_{0}\right|^{2}-c\right)}
$$

We then justify the semi-classical limit for the observables $\left(\rho^{\epsilon}, u^{\epsilon}\right)$ connected with these global solutions and we conclude by verifying their WKB expansions.

We comment in passing on two related difficulties associated with justifying the global semi-classical limit in this case. First, the QEP equations represent a dispersive regularization of the limiting Euler-Poisson equations, and as such, they do not lend themselves to the standard energy method. Instead, the analysis of small dispersive limits usually proceeds by exploiting sufficiently many integrals of the motion. A case in point is the recent study of semi-classical limit of the defocusing cubic NLS carried out in [JLM2]. In our case, however, the nonlinearity of the dispersive correction yields only two such integrals that we know of - the total density and energy recorded in (2.1)-(2.2) below. Second, the regularity of the Euler Poisson equations with subcritical initial data is based on tracing along particle paths, the delicate balance between the focusing effect of convective terms and the repulsive Poisson forcing. We lack, however, the usual energy dissipation estimates in this case. Consequently, one should not expect to extend a classical energy approach, whose general framework in the context of quantum hydrodynamics can be found in [JLM1], in order to justify the semi-classical limit in the present setting of sub-critical NLSP problem.

To circumvent these difficulties, our analysis of the $2 \times 2$ QEP system (1.9)-(1.10) is carried out in terms of a modified Madelung's transformation of the form, consult [Gre],

$$
\psi^{\epsilon}(x, t)=A^{\epsilon}(x, t) e^{i \Phi^{\epsilon}(x, t) / \epsilon} .
$$

Here $A^{\epsilon}$ is a complex valued amplitude which takes into account the slowly varying part of the solution and $\Phi^{\epsilon}$ represents the fast scale. There is a one-to-one correspondence between these modified variables, $\left(q^{\epsilon}:=\Phi_{x}^{\epsilon}, A^{\epsilon}:=a^{\epsilon}+i b^{\epsilon}\right)$ and the observables $\left(\rho^{\epsilon}, u^{\epsilon}=S_{x}^{\epsilon}\right)$ connected with the usual representation, $\psi^{\epsilon}=\sqrt{\rho^{\epsilon}} \exp \left\{i S^{\epsilon} / \epsilon\right\}$,

$$
\rho^{\epsilon}=\left|A^{\epsilon}\right|^{2}, \quad u^{\epsilon}=q^{\epsilon}+\epsilon \frac{a^{\epsilon} b_{x}^{\epsilon}-b^{\epsilon} a_{x}^{\epsilon}}{2 i \sqrt{\left(a^{\epsilon}\right)^{2}+\left(b^{\epsilon}\right)^{2}}}, \quad u^{\epsilon}:=S_{x}^{\epsilon}, q^{\epsilon}=: \Phi_{x}^{\epsilon} .
$$

This leads to an equivalent $3 \times 3$ modified system, (2.8)-(2.10), governing the modified velocity gradient $q^{\epsilon}$ and a slowly varying complex valued amplitude $A^{\epsilon}=a^{\epsilon}+i b^{\epsilon}$. Unlike the usual WKB variables, the governing equations for $U^{\epsilon}:=\left[\Phi_{x x}^{\epsilon}, a^{\epsilon}, b^{\epsilon}\right]$ form a strongly hyperbolic system, which enables us to bridge between the associated NLSP solution, $U^{\epsilon}$, and its semi-classical limit solution of the Euler-Poisson equations. 
The rest of the paper is summarized into main four theorems. We begin in $\S 2$ with the following local existence statement.

Theorem 1.1 (Local existence). Consider the NLSP equation (1.8) subject to a sequence of WKB initial data, $\psi_{0}^{\epsilon}(x)=A_{0}^{\epsilon}(x) \exp \left\{i \Phi_{0}^{\epsilon}(x) / \epsilon\right\}$, with $\left(\Phi_{0}^{\epsilon}(x), A_{0}^{\epsilon}(x)\right)$ uniformly bounded in $\left(H^{4}(\mathrm{IR}), H^{2}(\mathrm{IR})\right)$. Then there exists $T_{0}>0$,

$$
T_{0} \sim 1 /\left(\left\|\Phi_{0}^{\epsilon}\right\|_{H^{4}(\mathbb{R})}+\left\|A_{0}^{\epsilon}\right\|_{H^{2}(\mathbb{R})}\right),
$$

such that (1.8) admits a solution of the form

$$
\psi^{\epsilon}(x, t)=A^{\epsilon}(x, t) \exp \left\{i \Phi^{\epsilon}(x, t) / \epsilon\right\}, \quad 0 \leq t<T_{0} .
$$

Moreover $\quad\left(A^{\epsilon}(x, t), \Phi^{\epsilon}(x, t)\right) \quad$ remain $\quad$ bounded $\quad$ in $\quad L^{\infty}\left(\left[0, T_{0}\right], H^{2}(\mathbb{R})\right) \quad \times$ $L^{\infty}\left(\left[0, T_{0}\right], H^{4}(\mathrm{IR})\right)$ uniformly in $\epsilon$.

Theorem 1.1 tells us that the modified variables, $U^{\epsilon}:=\left[d^{\epsilon}:=\Phi_{x x}^{\epsilon}, a^{\epsilon}, b^{\epsilon}\right]$ converge to a limit, $U:=[d, a, b]$. This limit is governed by a modified limit system, (3.1)-(3.3), which is studied in $\S 3$. Our next result, outlined in $\S 4$, complements Theorem 1.1 with an error estimate for $\left\|U^{\epsilon}-U\right\|$.

TheOREM 1.2 (Error estimate). Consider the NLSP equation (1.8) subject to a sequence of WKB initial data $\psi_{0}^{\epsilon}(x)=A_{0}^{\epsilon}(x) \exp \left\{i \Phi_{0}^{\epsilon}(x) / \epsilon\right\}$, with $\left(\Phi_{0}^{\epsilon}(x), A_{0}^{\epsilon}(x)\right)$ uniformly bounded in $\left(H^{4}(\mathrm{IR}), H^{2}(\mathrm{IR})\right)$. Let $U^{\epsilon}=\left[d^{\epsilon} \equiv \Phi_{x x}^{\epsilon}, a^{\epsilon}, b^{\epsilon}\right], \quad t \leq T_{0}$ denote the modified variables associated with the local NLSP solution $A^{\epsilon}(x, t) \exp \left\{i \Phi^{\epsilon} / \epsilon\right\}, t \leq$ $T_{0}$, and let $U=[d, a, b]$ denote the solution of the modified limit system, consult (3.1)(3.3) below. Then $H^{2}-\lim U^{\epsilon}(\cdot, t)=U(\cdot, t), t \leq T_{0}$, and the following error estimate holds

$$
\left\|U^{\epsilon}(\cdot, t)-U(\cdot, t)\right\|_{H^{2}} \leq \text { Const. }\left[\left\|U_{0}^{\epsilon}(x)-U_{0}(x)\right\|_{H^{2}}+\epsilon\right], \quad t \leq T_{0} .
$$

The modified limit system (3.1)-(3.3) is equivalent with Euler-Poisson equations (1.11)-(1.12). In particular, the $H^{2}$-regularity of its limit solution, $U(\cdot, t)$ is dictated by the regularity of the Euler-Poisson equation. We now invoke the global regularity of Euler-Poisson solutions, established in [ELT] under the sub-critical condition (1.13). It follows that $\|U(\cdot, t)\|_{H^{2}}$ and hence $\left\|U^{\epsilon}(\cdot, t)\right\|_{H^{2}}$ remain bounded for $t \leq$ Const. $\ln \frac{1}{\epsilon}$ and we can therefore extend the local solution constructed above. The argument is classical - consult e.g., [Co] in the context of global Navier-Stokes solutions during the regularity interval of Euler equations. We conclude, in $\S 5$ with the following statement of global existence.

Theorem 1.3 (Global existence). Consider the NLSP equation (1.8) subject to WKB initial data, $\psi(x, 0)=A_{0}(x) \exp \left\{i \Phi_{0}(x) / \epsilon\right\}$, with $\Phi_{0}(x)=S_{0}(x) \in H^{4}(\mathrm{IR})$, and $A_{0}(x)=\sqrt{\rho_{0}(x)} \in H^{2}(\mathbb{R}), \int\left(\rho_{0}(\cdot)-c\right)=0$. Assume that the initial data satisfy the sub-critical condition

$$
\left|\partial_{x}^{2} S_{0}(\alpha)\right|<\sqrt{k\left(2 \rho_{0}(\alpha)-c\right)}, \quad \alpha \in \mathbb{R} .
$$

Then for any $T>0$, there exists $\epsilon_{0}=\epsilon_{0}(T)$ such that, for every $0<\epsilon \leq \epsilon_{0}$, the $N L S P$ equation (1.8) admits a global solution of the form $\psi^{\epsilon}=A^{\epsilon}(x, t) \exp \left\{i \Phi^{\epsilon}(x, t) / \epsilon\right\}$ for all $t<T$, where $\Phi_{x x}^{\epsilon}, A^{\epsilon}=a^{\epsilon}+i b^{\epsilon}$ are bounded in $L^{\infty}\left([0, T], H^{2}(\mathrm{IR})\right)$ uniformly in $\epsilon$. 
Moreover, its semi-classical limit $\left(d=\Phi_{x x}, a, b\right)$ is the global solution of the modified system (3.1)-(3.3) and the following error estimate holds

$$
\left\|\Phi_{x x}^{\epsilon}(\cdot, t)-\Phi_{x x}(\cdot, t)\right\|_{H^{2}}+\left\|A^{\epsilon}(\cdot, t)-A(\cdot, t)\right\|_{H^{2}} \leq \text { Const }_{T} \cdot \epsilon, \quad t \leq T .
$$

Finally, we are ready to convert the last global statement, yielding a complete global characterization of the semi-classical limit of the one-dimensional NLSP in terms of the usual observable variables, $\rho^{\epsilon}, u^{\epsilon}$. Relations (1.14) and Theorem 1.3 imply the $H_{2}$ convergence of $\rho^{\epsilon} \longrightarrow \rho(x, t)$ of order $\mathcal{O}(\epsilon)$. Moreover, we can use the fact that $H^{1}(\mathrm{IR})$ is an algebra to conclude that

$$
\epsilon\left\|\frac{a^{\epsilon} b_{x}^{\epsilon}-b^{\epsilon} a_{x}^{\epsilon}}{2 i \sqrt{\left(a^{\epsilon}\right)^{2}+\left(b^{\epsilon}\right)^{2}}}\right\|_{H^{1}} \leq \text { Const. } \epsilon
$$

and hence the $H_{1}$ convergence of $u^{\epsilon}=q^{\epsilon}+\mathcal{O}(\epsilon) \longrightarrow u$ of order $\mathcal{O}(\epsilon)$ follows. We conclude

Corollary 1.1 (Global convergence revisited). Consider the NLSP equation (1.8) subject to WKB initial data (1.7), $\psi_{0}^{\epsilon}(x)=\sqrt{\rho_{0}(x)} \exp \left\{i S_{0}(x) / \epsilon\right\}$, with $\left(\rho_{0}(x), S_{0}(x)\right)$ bounded in $\left(H^{2}(\mathrm{IR}), H^{4}(\mathrm{IR})\right), \int\left(\rho_{0}(\cdot)-c\right)=0$. Assume that the initial data satisfy the sub-critical condition

$$
\left|\partial_{x}^{2} S_{0}(\alpha)\right|<\sqrt{k\left(2 \rho_{0}(\alpha)-c\right)}, \quad \alpha \in \mathbb{R} .
$$

Then, for $\epsilon$ small enough, (1.8) admits a global solution of the form

$$
\psi^{\epsilon}=\sqrt{\rho^{\epsilon}(x, t)} \exp \left\{i S^{\epsilon}(x, t) / \epsilon\right\}
$$

for all $t<$ Const.ln $\frac{1}{\epsilon}$, with the following semi-classical limit, $\left(\rho^{\epsilon}, u^{\epsilon}:=S_{x}^{\epsilon}\right) \longrightarrow(\rho, u)$ where $(\rho, u)$ is the solution of the Euler-Poisson equations (1.5)(1.6). Moreover, the following error estimate holds

$$
\left\|\rho^{\epsilon}(\cdot, t)-\rho(\cdot, t)\right\|_{H_{2}}+\left\|u^{\epsilon}(\cdot, t)-u(\cdot, t)\right\|_{H_{1}} \leq \text { Const }_{T} \cdot \epsilon, \quad t \leq T \sim \ln \frac{1}{\epsilon} .
$$

The above results enable us to justify the WKB expansion.

TheOREM 1.4 (WKB expansion). Consider the NLSP equation (1.8) subject to sub-critical WKB initial data, $\psi(x, 0)=A(x, 0) \exp \{i \Phi(x, 0) / \epsilon\}$

$$
\left|\partial_{x}^{2} \Phi(\alpha, 0)\right|<\sqrt{k\left(2|A(\alpha, 0)|^{2}-c\right)} .
$$

Fix an integer $N \geq 0$, and assume that $\left[\Phi_{x x}(\cdot, 0), A(\cdot, 0)\right] \in H^{s}, s>2 N+2$. Then (1.8) admits a solution of the form $\psi^{\epsilon}=A^{\epsilon} \exp \left\{i \Phi^{\epsilon} / \epsilon\right\}$,

$$
\begin{aligned}
& \left.A^{\epsilon}(x, t)\right)=\sum_{j=0}^{N} A_{j}(x, t) \epsilon^{j}+o\left(\epsilon^{N}\right), \quad A_{j} \in H^{s-2 j}(\mathbb{R}), \\
& \left.\Phi^{\epsilon}(x, t)\right)=\sum_{j=0}^{N} \Phi_{j}(x, t) \epsilon^{j}+o\left(\epsilon^{N}\right), \quad \Phi_{j} \in H^{s-2 j+2}(\mathbb{R}) .
\end{aligned}
$$


This then yields the usual $N$-terms WKB expansion

$$
A^{\epsilon}(x, t) \exp \left\{i \Phi^{\epsilon}(x, t) / \epsilon\right\}=\sum_{j=0}^{N} \sqrt{\rho_{j}(x, t)} \epsilon^{j} \exp \left\{i S_{j}(x, t) / \epsilon\right\}+o\left(\epsilon^{N}\right), \quad t \leq T \sim N \ln \frac{1}{\epsilon}
$$

with $S_{j}$ and $\rho_{j}$ determined by the WKB method.

We conclude our Introduction by mapping yet another possible route to justify the semi-classical limit in the current setting of NLSP. In the first step, one justifies the passage from one-dimensional NLSP to the limiting one-dimensional Vlasov-Poisson equation,

$$
\partial_{t} f(x, \xi, t)+\xi \partial_{x} f(x, \xi, t)+V_{x} \partial_{\xi} f(x, \xi, t)=0 ; \quad V_{x x}(x, t)=k\left(\int_{\xi} f(x, \xi, t) d \xi-c\right) .
$$

This passage, administered through the Wigner transform, $f^{\epsilon}:=\left(\mathcal{W} \psi^{\epsilon}\right)(x, \xi, t)$, was rigorously justified in $[\mathrm{LP}]$ and $[\mathrm{MM}]$ for multidimensional problems in the mixed type case and was recently proved in the one space dimension for the general case in [ZZM]. The second step requires a rigorous justification of the passage from Vlasov-Poisson to Euler-Poisson equations. This passage is administered through the measure solution,

$$
f(x, \xi, t)=\rho(x, t) \delta(\xi-u(x, t)),
$$

whose first two moments form a renormalized solution of the Euler-Poisson, consult $[\mathrm{GM}]$. In particular, for sub-critical $\left(\rho_{0}, u_{0}\right)$, the Euler-Poisson solution $(\rho(\cdot, t), u(\cdot, t))$ remains smooth, [ELT] and it coincides with such renormalized solution, [GLM, Corollary 3.2].

2. Local convergence in time. We can show that there are two global invariants for the quantum Euler-Poisson equation

$$
\begin{aligned}
& M(t)=\int_{\mathbb{R}}\left(\rho^{\epsilon}-c\right) d x=M(0), \\
& E(t):=\int_{\mathbb{R}}\left(\frac{1}{2} \rho^{\epsilon}\left(u^{\epsilon}\right)^{2}+\frac{k}{2}\left(V_{x}^{\epsilon}\right)^{2}+\epsilon^{2}\left(\left(\sqrt{\rho^{\epsilon}}\right)_{x}\right)^{2}\right) d x=E(0) .
\end{aligned}
$$

Following Grenier [Gre], we use a modified Madelung's transform

$$
\psi^{\epsilon}=A^{\epsilon}(x, t) e^{i \Phi^{\epsilon}(x, t) / \epsilon}
$$

with a complex-valued 'amplitude', $A^{\epsilon}$, representing the slowly varying part of the solution and an argument $\Phi^{\epsilon}$ dictating the fast part of the solution. In the present context, this modified transformation plays a key role in the derivation of global (in time) hyperbolicity which in turn justifies the global semi-classical limit. The relation with the usual observables is revealed upon the change of variables

$$
\psi^{\epsilon}=\sqrt{\rho^{\epsilon}} e^{i S^{\epsilon} / \epsilon} \equiv\left|A^{\epsilon}\right| \exp \left\{i\left(S^{\epsilon}+\epsilon \arg A^{\epsilon}\right) / \epsilon\right\}
$$

which yields

$$
\begin{array}{r}
\rho^{\epsilon}:=\left|\psi^{\epsilon}\right|^{2}=\left|A^{\epsilon}\right|^{2}, \\
u^{\epsilon}:=S_{x}^{\epsilon}=q^{\epsilon}+\epsilon\left(\bar{A}^{\epsilon} A_{x}^{\epsilon}-A^{\epsilon} \bar{A}_{x}^{\epsilon}\right) /\left(2 i \rho^{\epsilon}\right), \quad q^{\epsilon}:=\Phi_{x}^{\epsilon} .
\end{array}
$$


Substitution of $\psi^{\epsilon}=A^{\epsilon} \exp \left\{i \Phi^{\epsilon} / \epsilon\right\}$ into the NLSP (1.8) leads to

$$
-i \epsilon A_{t}^{\epsilon}+\Phi_{t}^{\epsilon} A^{\epsilon}-\frac{\epsilon^{2}}{2} A_{x x}^{\epsilon}-i \epsilon \Phi_{x}^{\epsilon} A_{x}^{\epsilon}-\frac{i \epsilon}{2} A^{\epsilon} \Phi_{x x}^{\epsilon}+\frac{1}{2} A^{\epsilon}\left(\Phi_{x}^{\epsilon}\right)^{2}-V^{\epsilon} A^{\epsilon}=0
$$

with

$$
V_{x x}^{\epsilon}=\left|A^{\epsilon}\right|^{2}-c
$$

We split the above equation between the slowly varying part governed by the complex valued $A^{\epsilon}$, and the fast scale governed by $\Phi^{\epsilon}$,

$$
\begin{aligned}
& \Phi_{t}^{\epsilon}+\frac{1}{2}\left(\Phi_{x}^{\epsilon}\right)^{2}=V^{\epsilon}, \quad V_{x x}^{\epsilon}=k\left(\left|A^{\epsilon}\right|^{2}-c\right), \\
& A_{t}^{\epsilon}+\Phi_{x}^{\epsilon} A_{x}^{\epsilon}+\frac{1}{2} A^{\epsilon} \Phi_{x x}^{\epsilon}-\frac{i \epsilon}{2} A_{x x}^{\epsilon}=0 .
\end{aligned}
$$

Setting the velocity $q^{\epsilon}=\Phi_{x}^{\epsilon}$ and the electric charge, $e^{\epsilon}:=V_{x}^{\epsilon} / k$, gives

$$
\begin{aligned}
& q_{t}^{\epsilon}+q^{\epsilon} q_{x}^{\epsilon}=k e^{\epsilon}, \quad e_{x}^{\epsilon}=\left|A^{\epsilon}\right|^{2}-c, \\
& A_{t}^{\epsilon}+q^{\epsilon} A_{x}^{\epsilon}+\frac{1}{2} A^{\epsilon} q_{x}^{\epsilon}=\frac{i \epsilon}{2} A_{x x}^{\epsilon} .
\end{aligned}
$$

Separating the real and the imaginary part of $A^{\epsilon}:=a^{\epsilon}+i b^{\epsilon}$, the amplitude equation reads

$$
\begin{aligned}
& a_{t}^{\epsilon}+q^{\epsilon} a_{x}^{\epsilon}+\frac{1}{2} a^{\epsilon} q_{x}^{\epsilon}=-\frac{\epsilon}{2} b_{x x}^{\epsilon} \\
& b_{t}^{\epsilon}+q^{\epsilon} b_{x}^{\epsilon}+\frac{1}{2} b^{\epsilon} q_{x}^{\epsilon}=\frac{\epsilon}{2} a_{x x}^{\epsilon}
\end{aligned}
$$

To justify the uniform estimates of $\Phi^{\epsilon}$ and $A^{\epsilon}$, the velocity is dealt in terms of its derivative, $d^{\epsilon}:=q_{x}^{\epsilon}=\Phi_{x x}^{\epsilon}$, satisfying the transport equation

$$
d_{t}^{\epsilon}+q^{\epsilon} d_{x}^{\epsilon}+\left(d^{\epsilon}\right)^{2}=k e_{x}^{\epsilon}=k\left(\left|a^{\epsilon}+i b^{\epsilon}\right|^{2}-c\right) .
$$

We arrive at the following system

$$
\begin{aligned}
& d_{t}^{\epsilon}+q^{\epsilon} d_{x}^{\epsilon}=k\left(\left|a^{\epsilon}+i b^{\epsilon}\right|^{2}-c\right)-\left(d^{\epsilon}\right)^{2} \\
& a_{t}^{\epsilon}+q^{\epsilon} a_{x}^{\epsilon}=-\frac{1}{2} a^{\epsilon} d^{\epsilon}-\frac{\epsilon}{2} b_{x x}^{\epsilon} \\
& b_{t}^{\epsilon}+q^{\epsilon} b_{x}^{\epsilon}=-\frac{1}{2} b^{\epsilon} d^{\epsilon}+\frac{\epsilon}{2} a_{x x}^{\epsilon}
\end{aligned}
$$

We observe that even when the modified variables assume initial data with a real amplitude, $\left(a^{\epsilon}(x, 0), b^{\epsilon}(x, 0)\right)=\left(\sqrt{\rho_{0}^{\epsilon}}, 0\right)$, the evolution of $(2.10)$ still develops an imaginary component, $b^{\epsilon}(\cdot, t) \neq 0$.

The system (2.8)-(2.10) can be written in the compact form in terms of the 3vector $U^{\epsilon}:=\left[d^{\epsilon}, a^{\epsilon}, b^{\epsilon}\right]^{\top}$

$$
U_{t}^{\epsilon}+q^{\epsilon} U_{x}^{\epsilon}=\frac{\epsilon}{2} J U_{x x}^{\epsilon}+Q\left(U^{\epsilon}, U^{\epsilon}\right), \quad J:=\left[\begin{array}{ccc}
0 & 0 & 0 \\
0 & 0 & -1 \\
0 & 1 & 0
\end{array}\right]
$$


with a quadratic nonlinear term given by

$$
Q\left(U^{\epsilon}, U^{\epsilon}\right)=\left[\begin{array}{c}
k\left(\left|a^{\epsilon}+i b^{\epsilon}\right|^{2}-c\right)-\left(d^{\epsilon}\right)^{2} \\
-a^{\epsilon} d^{\epsilon} / 2 \\
-b^{\epsilon} d^{\epsilon} / 2
\end{array}\right] .
$$

Observing that (2.11) is a symmetric hyperbolic system, will enable us to proceed in the usual fashion with classical energy estimate. We use $(\cdot, \cdot),\|\cdot\|$ to denote the usual $L^{2}$ products and norms, and we seek a short time uniform bound of $\left\|U^{\epsilon}\right\|_{m}^{2}:=$ $\sum_{l \leq m}\left\|\partial_{x}^{l} U^{\epsilon}\right\|_{L^{2}}^{2}$. Differentiation of (2.11) with respect to $x$ leads to

$$
\left(\partial_{x}^{m} U^{\epsilon}\right)_{t}+\partial_{x}^{m}\left(q^{\epsilon} U_{x}^{\epsilon}\right)=\frac{\epsilon}{2} J\left(\partial_{x}^{m} U^{\epsilon}\right)_{x x}+\partial_{x}^{m} Q\left(U^{\epsilon}, U^{\epsilon}\right), \quad m=0,1,2 .
$$

Taking the inner product of the above equation with $\partial_{x}^{m} U^{\epsilon}$ with $m=2$ yields

$$
\begin{aligned}
\frac{1}{2} \frac{d}{d t}\left\|\partial_{x}^{2} U^{\epsilon}\right\|^{2} & =\left(\partial_{t} \partial_{x}^{2} U^{\epsilon}, \partial_{x}^{2} U^{\epsilon}\right) \\
& =\left(-\partial_{x}^{2}\left(q^{\epsilon} U^{\epsilon}\right)_{x}, \partial_{x}^{2} U^{\epsilon}\right)+\frac{\epsilon}{2}\left(J\left(\partial_{x}^{2} U^{\epsilon}\right)_{x x}, \partial_{x}^{2} U^{\epsilon}\right)+\left(\partial_{x}^{2} Q\left(U^{\epsilon}, U^{\epsilon}\right), \partial_{x}^{2} U^{\epsilon}\right) \\
& =: I_{1}+I_{2}+I_{3} .
\end{aligned}
$$

For the first term we have

$$
I_{1}=\left(-\partial_{x}^{2}\left(q^{\epsilon} U^{\epsilon}\right)_{x}, \partial_{x}^{2} U^{\epsilon}\right)=-\sum_{k=0}^{2}\left(\begin{array}{l}
2 \\
k
\end{array}\right)\left(\partial_{x}^{k} q^{\epsilon} \partial_{x}^{3-k} U^{\epsilon}, \partial_{x}^{2} U^{\epsilon}\right)=: \sum_{k=0}^{2} I_{1 k} .
$$

Integration by parts yields $\left|I_{10}\right|=\left|\left(q^{\epsilon} \partial_{x}^{3} U^{\epsilon}, \partial_{x}^{2} U^{\epsilon}\right)\right| \equiv\left|\frac{1}{2}\left(q_{x}^{\epsilon} \partial_{x}^{2} U^{\epsilon}, \partial_{x}^{2} U^{\epsilon}\right)\right| \leq$ $\left\|d^{\epsilon}\right\|_{\infty}\left\|U^{\epsilon}\right\|_{2}^{2}$. For the other two terms we have

$$
\begin{aligned}
& \left|I_{11}\right|=2\left|\left(q_{x}^{\epsilon} \partial_{x}^{2} U^{\epsilon}, \partial_{x}^{2} U^{\epsilon}\right)\right| \leq\left\|d^{\epsilon}\right\|_{\infty}\left\|U^{\epsilon}\right\|_{2}^{2}, \\
& \left|I_{12}\right|=\left|\left(q_{x x}^{\epsilon} \partial_{x} U^{\epsilon}, \partial_{x}^{2} U^{\epsilon}\right)\right| \leq\left\|d_{x}^{\epsilon}\right\|_{\infty}\left\|U^{\epsilon}\right\|_{2}^{2},
\end{aligned}
$$

and together with one-dimensional Sobolev estimate, the last three upper bounds imply that

$$
\left|I_{1}\right| \leq \text { Const. }\left(\left\|d^{\epsilon}\right\|_{\infty}+\left\|d_{x}^{\epsilon}\right\|_{\infty}\right)\left\|U^{\epsilon}\right\|_{2}^{2} \leq \text { Const. }\left\|U^{\epsilon}\right\|_{2}^{3} .
$$

Integration by parts and the anti-symmetry of $J$ show that the dispersive term vanishes,

$$
I_{2}=\frac{\epsilon}{2}\left(J\left(\partial_{x}^{2} U^{\epsilon}\right)_{x x}, \partial_{x}^{2} U^{\epsilon}\right)=-\frac{\epsilon}{2}\left(J \partial_{x}^{3} U^{\epsilon}, \partial_{x}^{3} U^{\epsilon}\right)=0 .
$$

We are left with the last quadratic term

$$
\begin{aligned}
I_{3} & =\left(\partial_{x}^{2} Q\left(U^{\epsilon}, U^{\epsilon}\right), \partial_{x}^{2} U^{\epsilon}\right) \\
& =\left(\left(k\left(\left|a^{\epsilon}+i b^{\epsilon}\right|^{2}-c\right)-\left(d^{\epsilon}\right)^{2}\right)_{x x}, d_{x x}^{\epsilon}\right)-\frac{1}{2}\left(\left(a^{\epsilon} d^{\epsilon}\right)_{x x}, a_{x x}^{\epsilon}\right)-\frac{1}{2}\left(\left(b^{\epsilon} d^{\epsilon}\right)_{x x}, b_{x x}^{\epsilon}\right) \\
& =: I_{31}+I_{32}+I_{33} .
\end{aligned}
$$

Some further calculations imply that each of the last three expressions is upper bounded by Const. $\left\|U^{\epsilon}\right\|_{2}^{3}$; for example

$$
\left|I_{31}\right| \leq \frac{1}{2}\left\|d^{\epsilon}\right\|_{\infty}\left\|a^{\epsilon}\right\|_{2}^{2}+\left\|d_{x}^{\epsilon}\right\|_{\infty}\left\|a_{x}^{\epsilon}\right\| \cdot\left\|a_{x x}^{\epsilon}\right\|+\frac{1}{2}\left\|a^{\epsilon}\right\|_{\infty}\left\|d_{x x}^{\epsilon}\right\| \cdot\left\|a_{x x}^{\epsilon}\right\| \leq \text { Const. }\left\|U^{\epsilon}\right\|_{2}^{3},
$$


with similar estimates for $I_{32}$ and $I_{33}$. The estimate for $\left\|\partial_{x}^{m} U^{m}\right\|$ with $m=0,1$ can be similarly obtained. A combination of the above facts yields the usual Ricatti's equation

$$
\frac{d}{d t}\left\|U^{\epsilon}\right\|_{2} \leq C_{0}\left\|U^{\epsilon}\right\|_{2}^{2}
$$

The energy bound independent of $\epsilon$ asserted in Theorem 1.1 follows for a local time interval, $\left[0, T_{0}\right]$,

$$
\left\|U^{\epsilon}(\cdot, t)\right\|_{2} \leq 2\left\|U_{0}^{\epsilon}\right\|_{2}, \quad t \leq T_{0}:=\frac{1}{2 C_{0}\left\|U_{0}^{\epsilon}\right\|_{2}} .
$$

3. The modified limit system. To study the global convergence rate of the QEP system (1.9)-(1.10), we consider the modified Madelung transformation which led to the system of three equations (2.8)-(2.10). The corresponding $3 \times 3$ limit system takes of the form

$$
\begin{aligned}
d_{t}+q d_{x} & =k\left(|a+i b|^{2}-c\right)-d^{2}, \\
a_{t}+q a_{x} & =-\frac{1}{2} a d, \\
b_{t}+q b_{x} & =-\frac{1}{2} b d,
\end{aligned}
$$

with $q(\cdot, t)$ standing for the primitive of $d(\cdot, t)$ satisfying

$$
q_{t}+q q_{x}=k e, \quad e_{x}=|a+i b|^{2}-c .
$$

This modified system shares the same regularity properties as the $2 \times 2$ limit EulerPoisson system, (1.11-(1.12). This is the content of

LEMma 3.1 (Regularity). Let $T^{*}$ be the maximal time interval of regularity for the solution $U=(d, a, b)^{\top}$ of the modified system (3.1)-(3.4). Let $T$ be the maximal time of regularity for the solution $(\rho, u)^{\top}$ of the Euler-Poisson equation (1.11)-(1.12), subject to initial conditions $\left(\rho_{0}=a_{0}^{2}+b_{0}^{2}, u_{0}\right)^{\top}$. Then we have $T=T^{*}$.

Proof. Assume $(d, a, b)^{\top}$ is a smooth solution of the modified system (3.1)-(3.3), with $q$ denoting the velocity, $d=q_{x}$. A simple manipulation, $(3.2) \times 2 a+(3.3) \times 2 b$ yields that $\eta:=a^{2}+b^{2}$ satisfies the mass equation (1.11),

$$
\eta_{t}+(\eta q)_{x}=0, \quad \eta:=a^{2}+b^{2} .
$$

Spatial integration of (3.1) implies that the primitive $q=\int d$ satisfies

$$
q_{t}+q q_{x}=V_{x}, \quad V_{x x}=k\left(|a+i b|^{2}-c\right)=k(\eta-c) .
$$

We conclude that $(\eta, q)^{\top}$ is a smooth solution of Euler-Poisson system (1.11)-(1.12), subject to initial conditions $\left(\eta_{0}, q_{0}\right)^{\top}=\left(\rho_{0}, u_{0}\right)^{\top}$, Thus, by uniqueness, the two solutions coincide, $\left(\eta=a^{2}+b^{2}, q\right)^{\top}=(\rho, u)^{\top}$ for $t \leq T^{*}$ and hence $T^{*} \leq T$.

Conversely, assume that $(\rho, u)^{\top}$ is the smooth solution of Euler-Poisson system (1.11)-(1.12) for $t \in[0, T]$, subject to initial data $\left(\rho_{0} \geq 0, u_{0}\right)$ such that $\rho_{0}=a_{0}^{2}+b_{0}^{2}$. By assumption, $d=u_{x}$ is smooth. Consequently, for $t \in[0, T]$ one obtains smooth 
solutions $a$ and $b$ of the linear transport equations, (3.2) and (3.3), subject to initial conditions $\left(a_{0}, b_{0}\right)^{\top}$. Combining the two equations for $a$ and $b$ gives, as before

$$
\left(a^{2}+b^{2}\right)_{t}+q\left(a^{2}+b^{2}\right)_{x}=-\left(a^{2}+b^{2}\right) d .
$$

Subtracting this from the mass equation (1.11), we find that $w:=\rho-\left(a^{2}+b^{2}\right)$ is a solution of the transport equation

$$
w_{t}+u w_{x}=-w d,
$$

with zero initial data, $w(x, 0) \equiv 0$, and hence $w \equiv 0$, i.e., $\rho=a^{2}+b^{2}$. Differentiation of (1.12) then yields

$$
\left(\partial_{x} u\right)_{t}+u\left(\partial_{x} u\right)_{x}=-k(\rho-c)=-k\left(|a+i b|^{2}-c\right),
$$

which shows that $\left(\partial_{x} u, a, b\right)^{\top}$ is a smooth solution of the modified system (3.1)-(3.3) with initial data $\left(d_{0}, a_{0}, b_{0}\right)$ for $t \in[0, T]$ and hence $T \leq T^{*}$.

Lemma 3.1 tells us that the regularity of the extended system (3.1)-(3.4) is dictated by the regularity of the associated 1D model (1.11)-(1.12). The critical threshold of the latter was analyzed in [ELT], and the result is recorded here for the reader's convenience.

TheOREM 3.1 (Global regularity with sub-critical data). The system of EulerPoisson equations (1.11)-(1.12) admits a global smooth solution for sub-critical initial configurations $\left(\rho_{0}, u_{0}\right)$ with $\int_{\mathbb{R}}\left(\rho_{0}-c\right) d x=0$ such that

$$
\left|\partial_{x} u_{0}(\alpha)\right|<\sqrt{k\left(2 \rho_{0}(\alpha)-c\right)}, \quad \forall \alpha \in \mathbb{R} .
$$

The precise behavior of the solution of (1.11)-(1.12) is given along the particle paths

$$
x(\alpha, t):=\alpha+\frac{u(\alpha, 0)}{\sqrt{c k}} \sin (\sqrt{c k} t)+\frac{\int_{-\infty}^{x}\left(\rho_{0}-c\right) d x}{c}(1-\cos (\sqrt{c k} t) .
$$

If (3.5) holds then $x_{\alpha} \neq 0$, and we have

$\rho(x(\alpha, t), t)=\frac{\rho_{0}}{x_{\alpha}(\alpha, t)}, \quad u(x(\alpha, t), t)=u_{0}(\alpha) \cos (\sqrt{c k} t)+\frac{k \int_{-\infty}^{x}\left(\rho_{0}-c\right) d x}{\sqrt{c k}} \sin (\sqrt{c k} t)$.

If condition (3.5) fails, then the solution breaks down at the first finite time, $t_{c}$, where $x_{\alpha}\left(\alpha, t_{c}\right)$ vanishes.

Lemma 3.1 together with Theorem 3.1 yield

Theorem 3.2 (Critical threshold for modified system). The limit system (3.1)(3.4) with initial data $\left(d_{0}, a_{0}, b_{0}\right)^{\top}$ with $\int_{\mathbb{R}}\left(a_{0}^{2}+b_{0}^{2}-c\right) d x=0$ admits a globally smooth solution if and only if

$$
\left|d_{0}(\alpha)\right|<\sqrt{k\left(2 a_{0}^{2}(\alpha)+2 b_{0}^{2}(\alpha)-c\right)}, \quad \forall \alpha \in \mathbb{R}
$$

In this case the solution of (3.1)-(3.3) is given by

$$
\begin{aligned}
\left(a^{2}+b^{2}\right)(x(\alpha, t), t) & =\frac{a_{0}^{2}(\alpha)+b_{0}^{2}(\alpha)}{x_{\alpha}(\alpha, t)}, \\
d(x(\alpha, t), t) & =\frac{d_{0}(\alpha) \cos (\sqrt{c k} t)+\sqrt{k / c}\left(\rho_{0}(\alpha)-c\right) \sin (\sqrt{c k} t)}{x_{\alpha}(\alpha, t)} .
\end{aligned}
$$


4. Local convergence rate. According to Theorem 1.1, the modified QEP solution $U^{\epsilon}=\left(d^{\epsilon}, a^{\epsilon}, b^{\epsilon}\right)^{\top}$ remains uniformly bounded together with couple of its derivatives, independent of $\epsilon$. Passing to the limit for the time interval $t \leq T_{0}$, the QEP solution converges to a limit solution, $U$, which is governed by the EulerPoisson equations in their modified formulation (3.1)-(3.3). We want to estimate the convergence error. To prove Theorem 1.2 let us consider the difference $W^{\epsilon}:=U^{\epsilon}-U$ which is governed by the error equation

$$
W_{t}^{\epsilon}+q^{\epsilon} W_{x}^{\epsilon}=\left(q-q^{\epsilon}\right) U_{x}+\frac{\epsilon}{2} J W_{x x}^{\epsilon}+\frac{\epsilon}{2} J U_{x x}+Q\left(U^{\epsilon}, U^{\epsilon}\right)-Q(U, U) .
$$

We seek an error bound $\left\|\partial_{x}^{2} W^{\epsilon}\right\|^{2}$. A straightforward energy method yields

$$
\begin{aligned}
\frac{1}{2} \frac{d}{d t}\left\|\partial_{x}^{2} W^{\epsilon}\right\|^{2}= & \left(\partial_{x}^{2} \partial_{t} W^{\epsilon}, \partial_{x}^{2} W^{\epsilon}\right) \\
= & \left(-\partial_{x}^{2}\left(q^{\epsilon} \partial_{x} W^{\epsilon}\right), \partial_{x}^{2} W^{\epsilon}\right)+\left(\partial_{x}^{2}\left(\left(q^{\epsilon}-q\right) \partial_{x} U\right), \partial_{x}^{2} W^{\epsilon}\right) \\
& \left.+\frac{\epsilon}{2}\left(J \partial_{x}^{2}\left(U_{x x}+W_{x x}^{\epsilon}\right)\right), \partial_{x}^{2} W^{\epsilon}\right)+\left(\partial_{x}^{2}\left(Q\left(U^{\epsilon}, U^{\epsilon}\right)-Q(U, U)\right), \partial_{x}^{2} W^{\epsilon}\right) \\
= & : I_{1}+I_{2}+I_{3}+I_{4} .
\end{aligned}
$$

For the first term we have the same estimate we had before for $I_{1}$ in (2.12)

$$
\left|I_{1}\right|=\left|\left(\partial_{x}^{2}\left(q^{\epsilon} W^{\epsilon}\right), \partial_{x}^{2} W^{\epsilon}\right)\right| \leq \text { Const. }\left\|U^{\epsilon}\right\|_{2}\left\|W^{\epsilon}\right\|_{2}^{2} .
$$

We now turn to the second term

$$
I_{2}=\left(\partial_{x}^{2}\left(\left(q^{\epsilon}-q\right) \partial_{x} U\right), \partial_{x}^{2} W^{\epsilon}\right)=\sum_{k=0}^{2}\left(\begin{array}{l}
2 \\
k
\end{array}\right)\left(\partial_{x}^{k}\left(q^{\epsilon}-q\right) \partial_{x}^{3-k} U, \partial_{x}^{2} W^{\epsilon}\right)=: \sum_{k=0}^{2} I_{2 k} .
$$

The expressions on the right are estimated from above by Sobolev inequality

$$
\begin{aligned}
& \left|I_{20}\right|=\left|\left(\left(q^{\epsilon}-q\right) \partial_{x}^{3} U, \partial_{x}^{2} W^{\epsilon}\right)\right| \leq\left\|\partial_{x}^{3} U\right\|_{\infty}\left\|q^{\epsilon}-q\right\|\left\|W^{\epsilon}\right\|_{2} \leq \text { Const. }\|U\|_{4}\left\|W^{\epsilon}\right\|_{2}^{2}, \\
& \left|I_{21}\right|=\left|2\left(\left(q^{\epsilon}-q\right)_{x} \partial_{x}^{2} U, \partial_{x}^{2} W^{\epsilon}\right)\right| \leq\left\|\partial_{x}^{2} U\right\|_{\infty}\left\|\left(q^{\epsilon}-q\right)_{x}\right\|\left\|W^{\epsilon}\right\|_{2} \leq \text { Const. }\|U\|_{3}\left\|W^{\epsilon}\right\|_{2}^{2}, \\
& \left|I_{22}\right|=\left|\left(\partial_{x}^{2}\left(q^{\epsilon}-q\right) U_{x}, \partial_{x}^{2} W^{\epsilon}\right)\right| \leq\left\|U_{x}\right\|_{\infty}\left\|q^{\epsilon}-q\right\|_{2}\left\|W^{\epsilon}\right\|_{2} \leq \text { Const. }\|U\|_{2}\left\|W^{\epsilon}\right\|_{2}^{2} .
\end{aligned}
$$

For the third term we have

$$
\left|I_{3}\right|=\epsilon\left|\left(J \partial_{x}^{2}\left(U_{x x}+W_{x x}^{\epsilon}\right), \partial_{x}^{2} W^{\epsilon}\right)\right|=\epsilon\left|\left(J \partial_{x}^{2} U_{x x}, \partial_{x}^{2} W^{\epsilon}\right)\right| \leq \epsilon\|U\|_{4}\left\|W^{\epsilon}\right\|_{2} .
$$

Finally, we treat the forth term, $I_{4}$. We first note that difference between the quadratics, $Q\left(U^{\epsilon}, U^{\epsilon}\right)-Q(U, U)$ must be of the form

$$
Q\left(U^{\epsilon}, U^{\epsilon}\right)-Q(U, U)=L\left[U,\left(U^{\epsilon}-U\right)\right]+R\left[\left(U^{\epsilon}-U\right), U^{\epsilon}\right],
$$

where $L$ and $R$ are some fixed bilinear form (which in this case involve the constant $k$ ). We split, accordingly, $I_{4}=J_{4}+K_{4}$ and we find

$$
J_{4}:=\left(\partial_{x}^{2} L\left[U, W^{\epsilon}\right], \partial_{x}^{2} W^{\epsilon}\right)=\sum_{k=0}^{2}\left(\begin{array}{c}
2 \\
k
\end{array}\right)\left(L\left[\partial_{x}^{k} U, \partial^{2-k} W^{\epsilon}\right], \partial_{x}^{2} W^{\epsilon}\right)
$$

and we find with the usual estimates

$$
\left|J_{4}\right| \leq \text { Const. } \sum_{k=0}^{2}\left\|\partial_{x}^{k} U\right\|_{\infty}\left\|W^{\epsilon}\right\|_{2-k}\left\|W^{\epsilon}\right\|_{2} \leq \text { Const }\|U\|_{3} \cdot\left\|W^{\epsilon}\right\|_{2}^{2} .
$$


A more careful treatment is required to handle $K_{4}$, when $L$ replaced by $R$,

$$
\begin{aligned}
K_{4} & :=\left(\partial_{x}^{2} R\left[W^{\epsilon}, U^{\epsilon}\right], \partial_{x}^{2} W^{\epsilon}\right) \\
& =\sum_{k=0}^{2}\left(\begin{array}{c}
2 \\
k
\end{array}\right)\left(R\left[\partial_{x}^{k} W^{\epsilon}, \partial^{2-k} U^{\epsilon}\right], \partial_{x}^{2} W^{\epsilon}\right)=: \sum_{k=0}^{2} K_{4 k},
\end{aligned}
$$

each of the terms on the right is upper bounded as follows,

$$
\begin{aligned}
& \left|K_{40}\right| \leq \text { Const. }\left\|W^{\epsilon}\right\|_{\infty}\left\|U^{\epsilon}\right\|_{2}\left\|W^{\epsilon}\right\|_{2} \leq \text { Const. }\left\|U^{\epsilon}\right\|_{2}\left\|W^{\epsilon}\right\|_{2}^{2}, \\
& \left|K_{41}\right| \leq \text { Const. }\left\|\partial_{x} W^{\epsilon}\right\|_{\infty}\left\|U^{\epsilon}\right\|_{1}\left\|W^{\epsilon}\right\|_{2} \leq \text { Const. }\left\|U^{\epsilon}\right\|_{2}\left\|W^{\epsilon}\right\|_{2}^{2}, \\
& \left|K_{42}\right| \leq \text { Const. }\left\|W^{\epsilon}\right\|_{2}\left\|U^{\epsilon}\right\|_{\infty}\left\|W^{\epsilon}\right\|_{2} \leq \text { Const. }\left\|U^{\epsilon}\right\|_{2}\left\|W^{\epsilon}\right\|_{2}^{2} .
\end{aligned}
$$

Summarizing the upper bound we have so far we conclude

$$
\frac{d}{d t}\left\|W^{\epsilon}\right\|_{2} \leq \epsilon\|U\|_{4}+C_{1}\left(\|U(\cdot, t)\|_{4}+\left\|U^{\epsilon}\right\|_{2}\right) \cdot\left\|W^{\epsilon}\right\|_{2} .
$$

According to Theorem 3.2, the particular solution $U$ and its derivatives remain uniformly bounded in time, since they could be expressed in terms of $\partial_{\alpha}^{k}\left(a^{2}+b^{2}\right), \partial_{\alpha}^{k} d$ and $\partial_{\alpha}^{k} u$ which are all periodic in time. Let $C_{4}$ denote the upper bound $\|U(\cdot, t)\|_{4} \leq C_{4}$. By Gronwall's inequality the error does not exceed

$$
\begin{aligned}
& \left\|U^{\epsilon}(\cdot, t)-U(\cdot, t)\right\|_{2} \\
\leq & \left\|U^{\epsilon}(\cdot, 0)-U(\cdot, 0)\right\|_{2}+C_{4} \epsilon \int_{s=0}^{t} \exp \left\{\int_{\tau=s}^{t} C_{1}\left(C_{4}+\| U^{\epsilon}\left(\cdot, \tau \|_{2}\right) d \tau\right\} d s .\right.
\end{aligned}
$$

We recall that for the short time interval, $t \leq T_{0}$, we have by Theorem 1.1, $\left\|U^{\epsilon}(\cdot, t)\right\|_{2} \leq 2\left\|U_{0}\right\|_{2}$ and (4.5) implies convergence rate of order $\mathcal{O}(\epsilon)$ asserted in Theorem 1.2.

5. Global convergence of semi-classical limit. The error equation (4.4) shows that during the local existence interval, $\left[0, T_{0}\right]$, the QEP solution cannot grow too fast as predicted by the pessimistic Ricatti bound (2.13),

$$
\frac{d}{d t}\left\|U^{\epsilon}\right\|_{2} \leq C_{0}\left\|U^{\epsilon}\right\|_{2}^{2} .
$$

Indeed, $U^{\epsilon}$ must stay within the $\mathcal{O}(\epsilon)$ neighborhood of the limit solution $U(\cdot)$, and in particular,

$\left\|U^{\epsilon}(\cdot, t)\right\|_{2} \leq\|U(\cdot, t)\|_{2}+\left\|U^{\epsilon}(\cdot, t)-U(\cdot, t)\right\|_{2} \leq C_{2}+C_{4} \epsilon \int_{s=0}^{t} e^{\int_{\tau=s}^{t} C_{1}\left(C_{4}+\| U^{\epsilon}\left(\cdot, \tau \|_{2}\right) d \tau\right.} d s$.

We want to extend this argument beyond the local existence interval $\left[0, T_{0}\right]$. The error bound (4.4) is valid for all $t$ 's, linking the growth of the error $e(t):=\left\|U^{\epsilon}(\cdot, t)-U(\cdot, t)\right\|_{2}$ to the growth of $\|U(\cdot, t)\|_{2}$,

$$
\frac{d}{d t} e(t) \leq C_{4} \epsilon+C_{1}\left(C_{4}+\left\|U^{\epsilon}\right\|_{2}\right) \cdot e(t) .
$$

Together with the upper bound $\left\|U^{\epsilon}\right\|_{2} \leq\|U\|_{2}+\left\|U^{\epsilon}(\cdot, t)-U(\cdot, t)\right\|_{2} \leq C_{2}+e(t)$ we conclude

$$
\frac{d}{d t} e(t) \leq C_{4} \epsilon+C_{1}\left(C_{4}+C_{2}+e(t)\right) \cdot e(t) \leq C\left[\epsilon+e(t)+e^{2}(t)\right], \quad e(0)=0 .
$$


This gives the inequality for $Z(t)=e(t) e^{-C t}$,

$$
\frac{d}{d t} Z(t) \leq C \epsilon e^{-C t}+C e^{C t} Z^{2}, \quad Z(0)=0 .
$$

Multiplying the above inequality by $\frac{\theta}{(1+\theta Z)^{2}}$ with $\theta$ chosen as

$$
\theta=\max \left\{4\left(e^{C T}-1\right), \frac{e^{C T}}{4 \epsilon\left(e^{C T}-1\right)}\right\},
$$

we obtain

$$
\frac{\theta Z^{\prime}}{(1+\theta Z)^{2}} \leq C \theta \epsilon e^{-C t}+C \theta^{-1} e^{C t}
$$

Integration over $[0, t]$ gives

$$
\frac{1}{1+\theta Z} \geq 1-\epsilon \theta\left(1-e^{-C t}\right)-\theta^{-1}\left(e^{C t}-1\right) \geq \frac{1}{2}
$$

for $\epsilon \leq \epsilon_{0}$ with $\epsilon_{0}=\frac{e^{C T}}{16\left(e^{C T}-1\right)^{2}}$ and $\theta$ chosen above. Therefore we have $Z \leq 1 / \theta$, i.e.,

$$
e(t) \leq \min \left\{\frac{e^{C T}}{4\left(e^{C T}-1\right)}, \quad 4 \epsilon\left(e^{C T}-1\right)\right\} .
$$

This shows that for any $T>0$, there exists a sufficiently small $\epsilon_{0} \sim e^{-C T}$, such that for $\epsilon \leq \epsilon_{0}$ we have $\left\|U^{\epsilon}(\cdot, t)-U(\cdot, t)\right\|_{2} \leq$ Const $_{T} \epsilon$ and Theorem 1.3 follows.

6. Validity of the WKB expansion. According to Theorem 1.3, the NLSP equation (1.8) admits a global solution with modified variables, $U^{\epsilon}=\left[\Phi_{x x}^{\epsilon}, a^{\epsilon}, b^{\epsilon}\right]$ such that $U^{\epsilon}=U+\mathcal{O}(\epsilon)$ where $U$ satisfies the limiting modified system (2.11). The latter is an hyperbolic system so that $U(\cdot, t)$ is in $H^{s}$ if $U(\cdot, 0)$ is. We want to show that the NLSP solution $\psi^{\epsilon}=A^{\epsilon} \exp \left\{i \Phi^{\epsilon} / \epsilon\right\}$, realized in terms of the 3-vector of modified variables $U^{\epsilon}:=\left[\Phi_{x x}^{\epsilon}, a^{\epsilon}, b^{\epsilon}\right]$, admits the following asymptotic expansion

$$
U^{\epsilon}=\sum_{j=0}^{N} U_{j} \epsilon^{j}+o\left(\epsilon^{N}\right), \quad U_{j} \in L^{\infty}\left([0, T], H^{s-2 j}(\mathbb{R})\right) .
$$

- Leading order

From Theorem 1.3 we know that $U^{\epsilon}$ are bounded in $L^{\infty}\left([0, T], H^{2}(\mathrm{IR})\right)$. Using the governing equation (2.11), $U_{t}^{\epsilon}$ are bounded in $L^{\infty}\left(0, T ; L^{2}(\mathbb{R})\right)$; in fact, according to (2.11), $U^{\epsilon}$ is given as the sum of three terms: an $H^{1} \times H^{1}$ term $q^{\epsilon} U_{x}^{\epsilon}$, a vanishing $L^{2}$ term, $\sim \epsilon J U_{x x}^{\epsilon}$ and an $H^{2}$ quadratic term, $Q\left(U^{\epsilon}, U^{\epsilon}\right)$, which are compact in $L^{2}$. It follows that a subsequence of $U^{\epsilon}$ converge uniformly in $L^{\infty}\left([0, T], L^{2}(\mathrm{IR})\right)$ to $U_{0}$, which are solutions of

$$
\partial_{t} U_{0}+q_{0} \partial_{x} U_{0}=Q\left(U_{0}, U_{0}\right), \quad U_{0}=\left[d_{0}, a_{0}, b_{0}\right], \quad d_{0}=\partial_{x} q_{0}=\partial_{x x} \Phi_{0}
$$

subject to $H^{s}$ initial data

$$
U_{0}(\cdot, 0)=\lim _{\epsilon \rightarrow 0} U^{\epsilon}(\cdot, 0) \in H^{s} .
$$

Hyperbolicity implies that $U_{0}(\cdot, t)$ remains in $H^{s}$ and the uniqueness of limit solution for this system ensures the convergence of the whole sequence, $U^{\epsilon} \longrightarrow$ $U_{0}$. 
- First order

To justify the first order term in the expansion, we consider the $H^{s-2}$-initial data

$$
U_{1}(\cdot, 0)=\lim _{\epsilon \rightarrow 0} \frac{U^{\epsilon}(\cdot, 0)-U_{0}(\cdot, 0)}{\epsilon} .
$$

Setting $W^{\epsilon}:=\left(U^{\epsilon}-U_{0}\right) / \epsilon$, and carrying out the energy estimates along the lines of $\S 4$ leads to an upper bound

$$
\left\|W^{\epsilon}(\cdot, t)\right\|_{s-2} \leq \mathcal{F}\left(\left\|U_{0}\right\|_{s}\right), \quad t \leq T,
$$

with a nonlinear dependence $\mathcal{F}$, depending on $\left\{\left\|U_{0}(\cdot, t)\right\|_{s}\right\}_{0 \leq t \leq T}$. Hence $W^{\epsilon}$ is bounded in $L^{\infty}\left([0, T], H^{s-2}\right)$, and $W_{t}^{\epsilon}$ is bounded in $L^{\infty}\left([0, T], H^{s-4}\right)$. As before, a subsequence, $W^{\epsilon}$ converges strongly in $L^{\infty}\left([0, T], H^{s-4}\right)$ to a limit function $U_{1}=\left[q_{1}, a_{1}, b_{1}\right]$. This limit solves the following first variation problem

$$
\partial_{t} U_{1}+q_{0} \partial_{x} U_{1}+q_{1} \partial_{x} U_{0}=Q\left(U_{0}, U_{0}\right)+\left[\begin{array}{ccc}
-2 d_{0} & 2 k a_{0} & 2 k b_{0} \\
-\frac{1}{2} a_{0} & -\frac{1}{2} d_{0} & 0 \\
-\frac{1}{2} b_{0} & 0 & -\frac{1}{2} d_{0}
\end{array}\right] U_{1},
$$

with initial data

$$
U_{1}(0)=\lim _{\epsilon \rightarrow 0} \frac{U^{\epsilon}(0)-U_{0}(0)}{\epsilon} .
$$

The hyperbolicity of $(6.1)$ implies that $U_{1}(\cdot, t)$ remains in $H^{s-2}$ and compact in $H^{s-4}$. The uniqueness of solution to this problem ensures the convergence of the whole sequence $W^{\epsilon}$ to $U_{1} \in H^{s-4}$.

- Higher order terms

Assume that we have already an asymptotic expansion to the order $N$

$$
U^{\epsilon}=\sum_{j=0}^{N} U_{j} \epsilon^{j}+o\left(\epsilon^{N}\right)
$$

with $U_{j} \in L^{\infty}\left([0, T], H^{s-2 j}\right)$. Let $W^{\epsilon} \equiv W_{N}^{\epsilon}$ denote the error

$$
W^{\epsilon}=\frac{U^{\epsilon}-\tilde{U}^{\epsilon}}{\epsilon^{N+1}}, \quad \tilde{U}^{\epsilon}:=\sum_{j=0}^{N} U_{j} \epsilon^{j}
$$

Inserted into (2.11), we find that $W^{\epsilon}$ satisfies

$$
\partial_{t} W^{\epsilon}+q^{\epsilon} \partial_{x} W^{\epsilon}+\left(q^{\epsilon}-\tilde{q}^{\epsilon}\right) \partial_{x} \tilde{U}^{\epsilon}=\frac{\epsilon}{2} J W_{x x}^{\epsilon}+\frac{1}{2 \epsilon^{N}} J \tilde{U}_{x x}^{\epsilon}+H^{\epsilon}\left(W^{\epsilon}, \tilde{U}^{\epsilon}\right)
$$

where $H^{\epsilon} \equiv H_{N}^{\epsilon}$ is given as the sum of bilinear form, consult (4.3)

$$
H_{N}^{\epsilon}=L\left(\tilde{U}^{\epsilon}, W^{\epsilon}\right)+R\left(\tilde{U}^{\epsilon}, W^{\epsilon}\right)+\epsilon^{N+1} Q\left(W^{\epsilon}, W^{\epsilon}\right) .
$$

One can show that $H_{N}^{\epsilon}(\cdot, t)$ is bounded $\left\|H_{N}\right\|_{s-2 N-2} \leq$ Const. $\left\|W^{\epsilon}\right\|_{s-2 N-2}^{2}$. Assume that the initial data satisfy

$$
\left\|W^{\epsilon}(0)\right\|_{s-2 N-2} \leq \text { Const. }
$$


then (6.2) implies that the scaled error, $e(t):=\| W\left(\cdot, t \|_{s-2 N-2}\right.$, satisfies $\dot{e}(t) \leq C_{2 s}+C_{1} e(t)+C_{2} e^{2}(t)$, which in turn yields

$$
\left\|W^{\epsilon}(\cdot, t)\right\|_{s-2 N-2} \leq \text { Const. } \quad t \leq T \sim N \ln \frac{1}{\epsilon} .
$$

We conclude that $\left\|U^{\epsilon}-\sum^{N} U_{j} \epsilon^{j}\right\|_{s-2 N-2} \leq \mathcal{O}\left(\epsilon^{N+1}\right)$.

- WKB expansion

The above results show that we have obtained the formal expansion of $A^{\epsilon}$ and $\Phi^{\epsilon}$ to an arbitrarily high order. To recover the usual WKB expansion, it suffices to equate the $\epsilon$-powers of the same order in the identity

$$
\sum_{j=0}^{\infty} \sqrt{\rho_{j}(x, t)} \epsilon^{j} e^{i S_{j}(x, t) / \epsilon}=\left(\sum_{m=0}^{\infty} A_{m} \epsilon^{m}\right) \exp \left(i \sum_{m=0}^{\infty} \Phi_{m} \epsilon^{m-1}\right) .
$$

Acknowledgments. Research was supported in part by ONR Grant No. N00014-91-J-1076(ET) and by NSF grant \#DMS01-07917 (ET, HL). We thank Peter A. Markowich for his interest and many valuable suggestions.

\section{REFERENCES}

[Co] P. Constantin, Note on loss of regularity for solutions of the 3-D incompressible Euler and related equations, Commun. Pure Appl. Math., 38 (1986), pp. 311-326.

[ELT] S. Engelberg, H. Liu And E. TAdmor, Critical Thresholds in Euler-Poisson Equations, Indiana Univ. Math. J., 50 (2001), pp. 109-157.

[GM] I. GASSER AND P. MARKOWICH, Quantum hydrodynamics, Wigner transforms and the classical limit, Asymptot. Anal., 14 (1997), pp. 97-116.

[GLM] I. Gasser, C.-K. Lin AND P. A. MARKOwich, A review of dispersive limits of nonlinear Schrödinger-type equations, Taiwanise J. Math., 4(4) (2000), pp. 501-529.

[Gre] E. Grenier, Semiclassical limit of the nonlinear Schrödinger equation in small time, Proc. Amer. Math. Soc., 126 (1998), pp. 523-530.

[JLM1] S. Jin, C.D. Levermore And D.W. MCLaughlin, The behavior of solutions of the NLS equation in the semiclassical limit, in: Singular Limits of Dispersive Waves, E. Ercolani, I. Gabitov, C.D. Levermore and D. Serre, eds., NATO ASI Series, Series B, Physics 320, Plenum, New York, 1994, pp. 235-256.

[JLM2] S. Jin, C.D. Levermore and D.W. McLaughlin, The semiclassical limit of the defocusing NLS hierarchy, Comm. Pure Appl. Math., 52 (1999), pp. 613-654.

[LL] L.D. LAndau And E.M. Lifschitz, Course of Theoretical Physics. Quantum Mechanics : Nonrelativistic Theory, vol. III, Oxford, Butterworth-Heinemann, 1976.

[LP] P.L. Lions And T. PAul, Sur les measures de Wigner, Rev. Mat. Iberoamericana, 9(9) (1993), pp. 553-618.

[MM] P. MARKOWICH AND N. MAUSER, The classical limit of a self-consistent quantum-Vlasov equation in 3-D, Math. Models Methods Appl. Sci., 3 (1993), pp. 109-124.

[SMM] C. SpARBER, P. MARKOWICH AND N. MAuser, Multivalued geometrical optics: Wigner functions versus WKB-methods, preprint (2001).

[ZZM] P. Zhang, Y. Zheng AND N. MAUseR, the limit from Schrodinger-Poisson to the VlasovPoisson equations with general data in one dimension, Comm. Pure Appl Math., LV (2002), pp. 582-632. 
H. LIU AND E. TADMOR 